\title{
Comparison of the Resistance of Bond Strength of Cemented Fiberglass Posts in Different Root Thirds with and without Silanization: An Ex Vivo Study
}

\author{
Gareth Robles ${ }^{1}$, Gustavo Huertas-Mogollón ${ }^{2}$, Yens Mendoza-Martiarena ${ }^{3}$, Gerardo Ayala ${ }^{4}$, Romel Watanabe ${ }^{5}$, \\ Daniel Alvitez-Temoche ${ }^{6}$, Frank Mayta-Tovalino ${ }^{7}$
}

\begin{abstract}
Aim: To compare the application of silane in the bond strength of fiberglass posts in different thirds of the cemented root with a self-adhesive system in an ex vivo study.

Materials and methods: Freshly extracted teeth were collected, which had indication of extraction for periodontal or orthodontic reasons. The study evaluated $n=42$ specimens that were divided into two large groups: I (control, without silane) and II (with silane); the resistance to adhesion in the respective thirds of the root (cervical, middle, and apical) was also evaluated. The prefabricated fiberglass posts were cemented with and without silane according to the manufacturer's instructions and were cemented with a resin-based cementing agent. The adhesion strength test was evaluated by the universal testing machine.

Results: According to the results of this experimental trial, the fiberglass posts that did not receive silane had the highest adhesion strength of $26.5 \pm 10.6 \mathrm{MPa}$, while silane-cemented posts only had 21.7 $\pm 8.1 \mathrm{MPa}$. The statistical analysis was performed using the Kruskal-Wallis test and work with a level of significance of $p<0.05$.

Conclusion: The use of silane as part of the cementing protocol in fiberglass posts does not influence the adhesion strength between the surfaces of the posts and the resin cement because there were statistically significant differences between both adhesion protocols.

Clinical significance: This study has a great impact because according to the results, clinical decisions can be made when cementing the fiberglass posts through the application of silane or not.

Keywords: Bond strength, Ex vivo study, Fiberglass post, Silane.

The Journal of Contemporary Dental Practice (2020): 10.5005/jp-journals-10024-2774
\end{abstract}

\section{INTRODUCTION}

The intraradicular posts have been indicated to guarantee an anchor and improve the conditions of the root remnant. ${ }^{1}$ Actually, the growing demand for oral esthetics has generated an interest in developing new systems of fiberglass posts which may cause high esthetics and adhesion. ${ }^{2,3}$ These fiber posts generally improve the visual effects of esthetic restorations ${ }^{4,5}$ and are widely indicated for the endodontic restoration of teeth; however, they can only be used when the remaining stump is tall enough to be reconstructed. ${ }^{6}$ The use of posts in cases where the structure of the teeth that have been destroyed by decay, trauma, or overly aggressive endodontic procedures is gaining wide acceptance among dentists. ${ }^{7}$

At present, an increase can be found in the literature on cementing protocols, adhesive systems, therefore, the clinician should be informed about the indications and uses of these prefabricated posts. ${ }^{8}$ Prefabricated posts consist of glass fibers embedded in a resinous matrix. ${ }^{9}$ It is known that the epoxy resin can bind composite materials based on resin and methacrylate through free radicals, ${ }^{4}$ which would allow cementation with adhesive resin. ${ }^{10,11}$ However, it is also known that the polymer matrix of the fiberglass posts does not react with adhesion, because the resinous cement has a high degree of conversion. ${ }^{12}$ Therefore, the adhesive cementation of the glass fiber posts is based on the binding of the silane to the post fibers.

Silanization is one of the most used techniques to generate a possible improvement in the conditions of adhesion. The silane-based agents have a molecule that is capable of reacting
1Postgraduate Department, School of Stomatology, Universidad
Católica Santo Toribio de Mogrovejo, Chiclayo, Peru
${ }^{2}$ Postgraduate Department, Faculty of Health Sciences, Universidad
Científica del Sur, Peru
${ }^{3-5}$ Academic Department of Rehabilitation Stomatology, Faculty of
Dentistry, Universidad Nacional Mayor de San Marcos, Peru
${ }^{6}$ Academic Department, Faculty of Dentistry, Universidad Nacional
Federico Villarreal, Peru
${ }^{7}$ Postgraduate Department, Faculty of Health Sciences, Universidad
Científica del Sur, Peru; Academic Department of Rehabilitation
Stomatology, Faculty of Dentistry, Universidad Nacional Mayor de San Marcos, Peru

Corresponding Author: Frank Mayta-Tovalino, Postgraduate Department, Faculty of Health Sciences, Universidad Científica del Sur, Peru; Academic Department of Rehabilitation Stomatology, Faculty of Dentistry, Universidad Nacional Mayor de San Marcos, Peru, Phone: +51 1 6106400, e-mail: fmaytat@ucientifica.edu.pe

How to cite this article: Robles G, Huertas-Mogollón G, MendozaMartiarena $Y$, et al. Comparison of the Resistance of Bond Strength of Cemented Fiberglass Posts in Different Root Thirds with and without Silanization: An Ex Vivo Study. J Contemp Dent Pract 2020;21 (3):261-266.

Source of support: Nil

Conflict of interest: None

with the inorganic filler of the fiberglass and the other with the resin. ${ }^{13}$ This mechanism of action causes the functional part and 
the inorganic content to bond. Improving the wettability of the back surface is another silanization effect. The highly cross-linked polymer matrix of the fiberglass posts is not reactive; ${ }^{14}$ therefore, only the fibers exposed on the surface of the prefabricated post can generate spaces for chemical bonding with silane molecules. The silane applied in restorative and esthetic dentistry is generally prehydrolyzed from monofunctional $\gamma$-methacryloxypropyltrimeth oxysilane which is diluted in a solution based on water and ethanol. ${ }^{4}$

Nowadays, the application of silane to improve the binding of resin-based luting agent to fiberglass poles is controversial. ${ }^{4}$ There is scientific literature that endorses that silanization does not have a significant effect on the adhesion resistance of resin cements on fiberglass posts. ${ }^{4,15,16}$ While other studies reported an increasing effect on adhesion strength through silanization. ${ }^{17}$

Therefore, the purpose of this ex vivo experimental study was to compare the resistance of bond strength of cemented fiberglass posts in different root thirds with and without silanization.

\section{Materials and Methods}

This research was an ex vivo experimental type, and the sample size was determined using the means comparison formula using the Stata ${ }^{\circledR}$ software version 15 , with an a of 0.05 and a power of 0.8 . The specimens $(n=42)$ were divided between each of the two subgroups (cervical, middle, and apical third) and formed the following groups (Fig. 1A):

Group I: Cemented fiberglass posts without silane (cervical)

Group II: Cemented fiberglass posts without silane (middle)

Group III: Cemented fiberglass posts without silane (apical)

Group IV: Cemented fiberglass posts with silane (cervical)

Group V: Cemented fiberglass posts with silane (middle)

Group VI: Cemented fiberglass posts with silane (apical)

In this study, the following criteria were used:

\section{Inclusion Criteria}

Single-root teeth, teeth without root fracture, teeth without dilated or curved root, and teeth with mature apex.

\section{Exclusion Criteria}

Teeth with attrition or erosion, teeth with cavities or cervical lesions, and teeth with short root.

\section{Preparation of Root Canals}

This research did not present ethical implications because an ex vivo experiment was performed with teeth extracted through an informed consent signed by each patient of said teeth that were extracted for reasons beyond the study, whether for orthodontic or periodontal reasons. A total of 42 dental pieces recently extracted were used for reasons beyond the development of this study (periodontal or orthodontic), which were totally unrelated to the objective of this study. These were placed in $0.2 \%$ sodium hypochlorite at $3^{\circ} \mathrm{C}$ for 3 months. The teeth were treated endodontically with the crown-down technique, in which Gates-Glidden (Union Broach, PA) \#2 diamond cutters were used and then rotary drills were used (ProFile 0.06 Taper Series 29; Dentsply Maillefer, Tulsa, OK). The teeth were irrigated between each instrumentation with $5.25 \%$ sodium hypochlorite with a $20-\mathrm{mL}$ syringe and a $23 \mathrm{G} 0.6 \mathrm{~mm} \times 25 \mathrm{~mm}$ blue hypodermic needle (Nipro). After root canal irrigation, the canal spaces were completely dried with absorbent paper cones (Dentsply Maillefer).
Then, the prepared canals were sealed with gutta-percha cones (Dentsply Maillefer) submerged in Grossman cement (Endo-G-Fill; Densell, New Zeland). After endodontic treatment, the teeth were guarded in $0.9 \%$ sodium chloride at $3^{\circ} \mathrm{C}$ for 7 days (Fig. 1B).

\section{Desobturation of Root Canals}

The crown of each specimen was sectioned perpendicular to the axial axis of the tooth $2 \mathrm{~mm}$ from the enamel-cement union with a $0.15 \times 22-\mathrm{mm}$ bi-diamond disk (KG Sorensen) by a low-speed straight micromotor at $40.000 \mathrm{rpm}$ (NSK EX-203C, Japan) and was irrigated during cutting with cold water. A working length of 13 $\mathrm{mm}$ was determined for the placement of the posts with an apical endodontic seal of 4-6 mm on all teeth. Subsequently, each root canal was prepared with an initial desobturation with Peeso 1 and 2 and then conformed with the Whitepost DC 1 corresponding to the diameter of the Whitepost DC 1 fiberglass post; all this was with a piece with counter-low-speed angle at 40.000 rpm and irrigation with $5.25 \%$ sodium hypochlorite.

\section{Insertion of Fiberglass Posts}

After the biomechanical preparation, the walls of the root canal were etched by the application of $37 \%$ phosphoric acid for 20 seconds and washed with distilled water during the same time and dried with paper absorbent cones. Then, the prepared specimens were randomly stored into two groups: control group for fiberglass posts without silane application (Whitepost DC 1; FGM) and experimental group for fiberglass posts with silane application (Whitepost DC 1; FGM) (Maquira, hydrolyzed 3 methacryloxypropyltrimethoxysilane). The insertion of the post was verified with the measure of $13 \mathrm{~mm}$ in each specimen thanks to a silicone stop inserted around the post (Fig. 1C).

\section{Cementation of Fiberglass Posts}

In the cementation of the posts without silane, the cementing resin (Relyx U200) was dosed and mixed in a ratio of 1:1 according to the instructions described by the manufacturer; the dual-cure resin cement was placed in the root canal using a lentulum, and the post was positioned inside the duct by a digital pressure for 10 seconds, then the excess cement was removed, then 6 minutes of self-curing was expected, and then light-curing with a light-emitting diode (LED) light lamp was carried out for 40 seconds (Woodpecker LED D Curing Light). In the cementation of the posts with silane, two layers of silane were applied on the fiberglass posts and allowed to dry for 3 minutes. Finally, the same cementation process was performed with Relyx U200 as was done with the group without silane (Fig. 1D).

\section{Preparation of Specimens}

The teeth were submerged in distilled water for 24 hours and stored at a temperature of $37^{\circ} \mathrm{C}$ until the millimeter cuts are made, according to ISO standards. To make the millimeter cuts, a lowspeed $0.15 \times 22$ - $\mathrm{mm}$ biactive diamond disk handpiece (KG Sorensen) was used by a low-speed straight micromotor at 40,000 rpm (NSK EX-203C) being irrigated during cutting with distilled water, and the roots were sectioned horizontally into three parts of $2 \mathrm{~mm}$ thickness corresponding to the cervical, middle, and apical thirds of the prefabricated post, thereby obtaining three samples per tooth of $2 \mathrm{~mm}$ thickness. The measurements were recorded using a Mitutoyo digital caliper (Mitutoyo Corp., Kanogawa, Japan). 

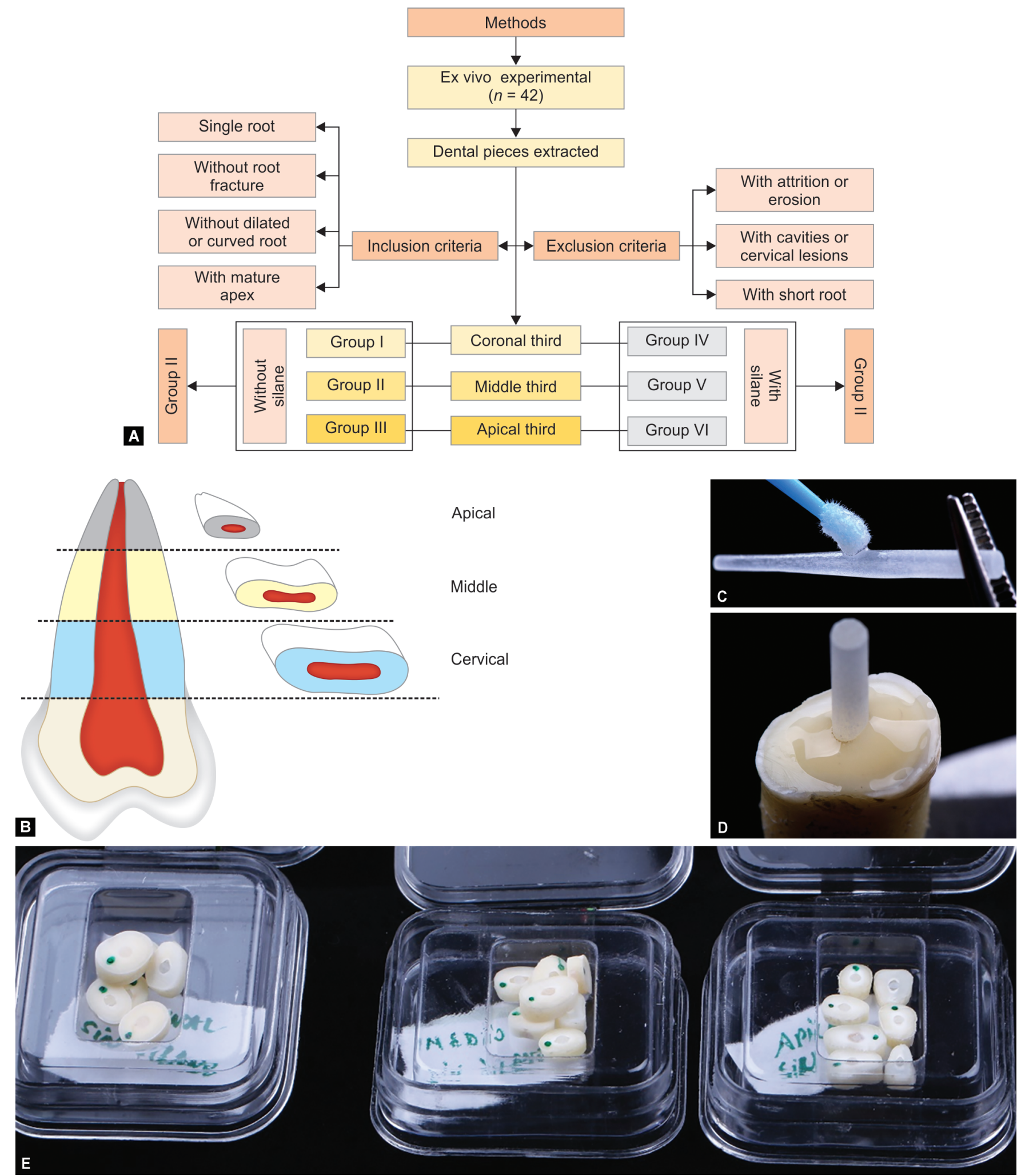

Figs 1A to E: (A) Sample selection flowchart; (B) Evaluation of bond strength in different parts (cervical, middle, and apical thirds); (C) Silane application on the fiberglass post; (D) Cementing of the fiberglass post with dual-cure resinous cement; (E) Root thirds after bond strength test

\section{Bond Strength Test}

The bond strength test was performed using the universal test machine LG WEW-300B brand. Data were collected according to the surface treatment performed on the post and on the root dentin.
The adhesive force (MPa) was calculated by dividing the tensile load (N) by the area $\left(\mathrm{mm}^{2}\right)$ of the adhesive interface of each specimen. Each tooth fragment was placed on the metal platform with the coronal surface facing down. The cylindrical tip of the machine was 
positioned only on the surface of the post, and it does not contact with the walls of the tooth segment. Then, the compressive force was applied with a load of 3-25 $\mathrm{kg}$ at a speed of $0.5 \mathrm{~mm} /$ minute. It was in an apical-coronal sense until the fragment of the post in the duct was detached. The values were recorded in kilogram-force per square millimeter which were then converted to megapascals to achieve the adhesive strength values where the diameters of each surface and the thickness were measured using a Mitutoyo digital pachymeter (Mitutoyo Corp.; Fig. 1E).

\section{Statistical Analysis}

For the univariate analysis, the descriptive statistics (mean and standard deviation) of the quantitative variables were obtained. Shapiro-Wilk test was used to determine the normality of the sample. Finally, for the comparison of groups, the non-probabilistic test of Kruskal-Wallis was used. A level of significance of $p<0.05$ was established. The data were analyzed with the statistical software Stata version 15 .

\section{Results}

\section{Adhesion of Silane-free Fiberglass Posts}

In the evaluation of the results obtained by regions in cemented posts without silane, the coronal third $(34.1 \pm 20.1 \mathrm{MPa})$ turned out to be the group with greater adhesion strength than the middle third $(32.1 \pm 9.2 \mathrm{MPa})$ and the apical third $(13.5 \pm 2.4 \mathrm{MPa})$. It is worth mentioning that in this group, only the coronal portion did not present a normal distribution ( $p=0.005$; Fig. 2 and Table 1).

\section{Adhesion of Fiberglass Posts with Silane}

When evaluating the results obtained by regions in the posts with silano, the coronal third $(30.2 \pm 7.8 \mathrm{MPa})$ turned out to be the group with greater adhesion strength than the middle third (18.1 $\pm 5.5 \mathrm{MPa})$ and the apical third $(16.9 \pm 11.5 \mathrm{MPa})$. However, in all the groups cemented with the use of silane, only the coronal and apical portions did not show a normal distribution ( $p<0.05$; Fig. 2 and Table 1).

\section{Comparison of Adhesion of Posts with and without Silane}

When comparing adhesion strength between regions that presented the fiberglass posts treated with and without silane application, it was found that there were statistically significant differences between the three portions (coronal, middle, and apical) cemented without silane $(p=0.001)$. Similarly, there were statistically significant differences in all portions of the silanecemented fiberglass pole group ( $p=0.027$; Fig. 2 and Table 1).

\section{Discussion}

Many investigations currently continue to demonstrate so much that silanization may or may not increase the effect of the bond strength at the surface level of the fiber posts; however, studies of greater scientific rigor are needed to confirm whether the application of silane significantly influences the resistance of adherence of prefabricated posts. This study provides the clinician with the theoretical bases that support whether or not it is necessary to use silane as a coupling agent on the surface of the fiberglass spikes before cementation, thus helping to reduce costs and working times. In this research, the influence of the chemical pretreatment with silane on the bond strength of the posts to the

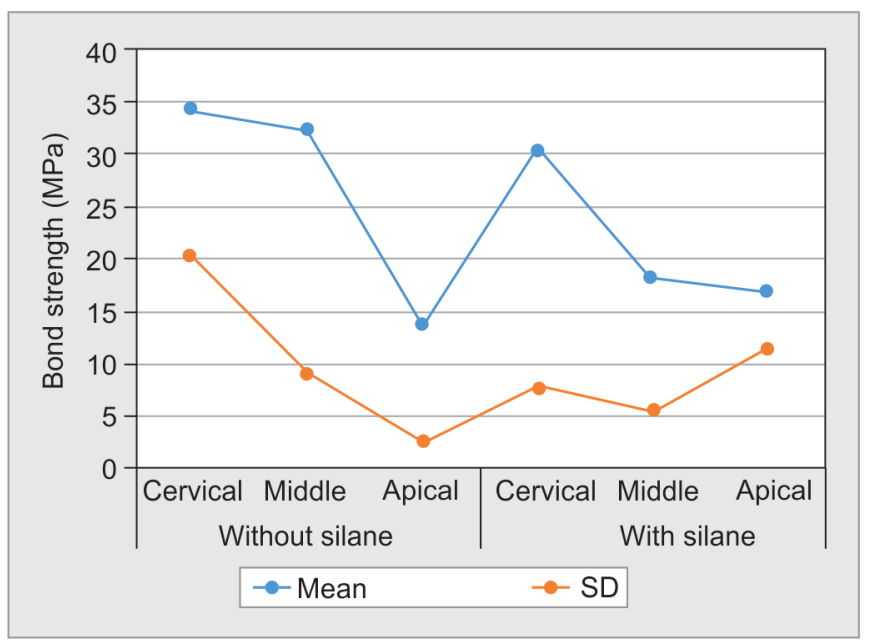

Fig. 2: Bond strength of the fiberglass posts with and without silane

self-etching and self-adhesive dual-cure resin cementation system was evaluated by means of a micropush-out test. ${ }^{18-20}$

The tensile test provides a more accurate estimate of adhesion resistance because the fracture occurs in parallel with the interface that simulates the conditions that occur in the clinical setting. ${ }^{19,21}$ The microtensilic test has the additional advantage of generating multiple "thin portions" in single-post samples. ${ }^{22}$ Therefore, the micropush-out test has been commonly accepted for adhesion strength assessment. ${ }^{19,20,23}$

However, because the cementation stabilizes the prefabricated post to the remaining stump, it is essential to assess the levels of adhesion in different root thirds. The variability of the dentinal tubules in size and number may be the possible explanation of why the adhesion varies according to the length of the root. It is known that the large-sized dentinal tubules are found in the coronal and middle thirds. ${ }^{24}$ Because the adhesion is increased through the penetration of the resinous cement in the dentinal tubules, the theory states that if there were a greater amount of dentinal tubules per square millimeter, stronger adhesion would be achieved. ${ }^{25}$ Another factor that can influence is the accessibility of the halogen light; generally, it does not enter until the apical thirds generate a possible limitation in the polymerization of the adhesive agents. ${ }^{4}$

In this study, the hydrolyzed 3-methacryloxypropyltrime thoxysilane coupling agent was employed; however, its application did not significantly show an increase in the adhesion resistance, compared with groups that did not receive silane, which shows the ineffectiveness of silanization that could be attributed to a weak chemical interaction. ${ }^{26}$

The role of the silane mainly lies in providing a chemical retention for the fiberglass posts through the chemical reaction with the adhesive matrix. ${ }^{4,26}$ Nonetheless, the finding in this study does not agree with the results of the previous studies. ${ }^{27-29}$ However, other studies contribute to the result obtained. ${ }^{4,26}$ That is why there is a great controversy about the efficiency of silanization of prefabricated posts to improve adhesion strength; this inconsistency in the results presented in the literature may be due to the differences in the composition of the resins used and the types of tests used. ${ }^{30}$

A clear example of this relationship is observed in some studies such as that of Cecchin et al., ${ }^{27}$ in which the highest bond strength was found for the silane and resin group without filler $(p<0.05)$. In 
Bond Strength of Cemented Fiberglass Posts in Different Root Thirds

Table 1: In vitro comparison of bond strength of posts with and without silane according to cemented root thirds

\begin{tabular}{llllccll}
\hline Group & Surface & Mean & Standard deviation & Minimum & Maximum & $p^{*}$ & $p^{* *}$ \\
\hline Without silane & Cervical & 34.1 & 20.1 & 20.7 & 3.1 & 0.005 & 0.001 \\
& Middle & 32.1 & 9.2 & 22.9 & 46 & 0.274 & \\
& Apical & 13.5 & 2.4 & 8.5 & 15.9 & 0.081 & \\
With silane & Cervical & 30.2 & 7.8 & 14.7 & 35.8 & 0.016 & 0.027 \\
& Middle & 18.1 & 5.5 & 10.6 & 27.5 & 0.949 & \\
& Apical & 16.9 & 11.5 & 8.4 & 40.4 & 0.011 \\
\hline
\end{tabular}

All values were recorded in megapascals

*Shapiro-Wilk test

**Kruskal-Wallis test

Level of significance, $p<0.05$

addition, the authors evidenced that the application of silane and resin without filler can improve the strength of the bond between the fiberglass post and the resin compound. However, the study by Pyun et al. ${ }^{28}$ which used another adhesion technique showed that the subsequent silanization of the fiberglass post and the heat treatment after $80^{\circ} \mathrm{C}$ with hot air blower can be beneficial in clinical cementation. However, etching with hydrogen peroxide before silanization was not effective according to the results shown. However, in contrast to some studies, the research by Belwalkar et al. ${ }^{29}$ differs since the authors found that silanization as a surface treatment did not improve the strength of the joint, but the synergism of presurface chemical treatments followed by silanization significantly improved the strength of the joint at the post-adhesive interface. This is consistent with the study by Archana et al., ${ }^{30}$ in which the surface treatment on the fiberglass pole by means of the silanization and sandblasting process significantly improved the adhesion between the pole and the resin cement interface. From the above, in our investigation, we also did not find a significant influence of silane on the adhesion in fiberglass posts for the restoration of endodontically treated teeth. Therefore, it is necessary to condition optimal adhesion on the post-root-cement interface for adequate longevity of future prosthetic restoration.

The scientific evidence shows that there are several methods to increase the adhesion of the fiberglass posts to the remaining dental tissue such as: dentin hybridization, placement of etching acid inside the root canal, acid conditioning in the walls of the root canal, use of self-adhesive cements, however all these factors should be studied in greater depth since the professional must observe the long-term clinical performance.

One of the main limitations of this ex vivo experimental research was that additional in vivo studies are needed to assess whether the performance of prefabricated fiberglass posts without silane treatment is similar to the performance found in an in vitro design. In addition, evidence is required to assess whether good adhesion is preserved by pretreating the surface of the fiberglass pole with the silane application before clinical use. However, the main importance of this study lies in the fact that it facilitates clinical operators to make decisions as to whether they should apply silane, saving operating time and money, because no statistically significant differences were found in the results of both the groups.

\section{Conclusion}

The following conclusions are made within the limitations of this ex vivo study: the use of silane did not significantly influence the bond strength between the surfaces of the fiberglass posts and the dual-cure resinous cement. Finally, the coronal third of the fiberglass post without the application of silane was more resilient to the root than the middle or apical third.

\section{Acknowledgments}

Authors wish to thank to Universidad Nacional de Ingeniería (UNI) and Universidad Científica del Sur, Lima, Peru, for their invaluable logistic support in the execution of the resistance to the adhesion of each of the specimens evaluated.

\section{References}

1. Stewardson DA. Non-metal post systems. Dent Updat 2001;28(7):326332. DOI: 10.12968/denu.2001.28.7.326.

2. Michalakis K, Hirayama H, Sfolkos S. Light transmission of posts and cores used for the anterior esthetic region. Int J Periodontics Restor Dent 2004;24(5):462-469. DOI: 10.11607/prd.00.0599.

3. Stefan P, Werder P. Clinical success of zirconium oxide posts with resin composite or glass ceramic cores in endodontically treated teeth: a 4-year retrospective study. Int J Prosthodont 2004;17(5):524-528.

4. Perdigao J, Gomes G, Lee IK. The effect of silane on the bond strengths of fiber posts. Dent Mater 2006;22(8):752-758. DOI: 10.1016/ j.dental.2005.11.002.

5. Demiryurek E, Kulunk S, Sarac D, et al. Effect of different surface treatments on the push-out bond strength of fiber post to root canal dentin. Oral Surg Oral Med Oral Pathol Oral Radiol Endod 2009;108:74-80. DOI: 10.1016/j.tripleo.2009.03.047.

6. Assif D, Gorfil C. Biomechanical considerations in restoring endodontically treated teeth. J Prosthet Dent 1994;71(6):565-567. DOI: 10.1016/0022-3913(94)90438-3.

7. Aksornmuang J, Foxton RM, Nakajima M, et al. Microtensile bond strength of a dual-cure resin core material to glass and quartz fibre posts. J Dent 2004;32(6):443-450. DOI: 10.1016/j.jdent.2004.03.001.

8. Pinheiro de Moraes A, Cenci M, Ratto de Moraes R, et al. Current concepts on the use and adhesive bonding of glass-fiber posts in dentistry: A review. Appl Adhes Sci 2013;1:1. DOI: 10.1186/2196-43511-1.

9. Mendoza D, Eakle W, Kahl E, et al. Root reinforcement with a resinbonded preformed post. J Prosthet Dent 1997;78(1):10-14. DOI: 10.1016/S0022-3913(97)70081-7.

10. Ferrari M, Vichi A, Grandini S. Efficacy of different adhesive techniques on bonding to root canal walls: An SEM investigation. Dent Mater 2001;17(5):422-429. DOI: 10.1016/S0109-5641(00)00102-0.

11. Sahmali S, Demirel F, Saygili G. Comparison of in vitro tensile bond strengths of luting cements to metallic and tooth-colored posts. Int J Periodontics Restor Dent 2004;24(3):256-263.

12. Bell A, Lassila L, Kangasniemi I, et al. Bonding of fibre-reinforced composite post to root canal dentin. J Dent 2005;33(7):533-539. DOI: 10.1016/j.jdent.2004.11.014. 
13. Matinlinna J, Lassila L, Ozcan M, et al. An introduction to silanes and their clinical applications in dentistry. Int J Prosthodont 2004;17(2):155-164.

14. Davis $\mathrm{P}$, Melo $L$, Foxton $\mathrm{R}$, et al. Flexural strength of glass fibrereinforced posts bonded to dual-cure composite resin cements. Eur J Oral Sci 2010;118(2):197-201. DOI: 10.1111/j.1600-0722.2010.00721.x.

15. Radovic I, Monticelli F, Goracci C, et al. The effect of sandblasting on adhesion of a dual-cured resin composite to methacrylic fiber posts: Microtensile bond strength and SEM evaluation. J Dent 2007;35(6):496-502. DOI: 10.1016/j.jdent.2007.01.009.

16. Bitter K, Meyer-Lueckel H, Priehn K, et al. Effects of luting agent and thermocycling on bond strengths to root canal dentine. Int Endod J 2006;39(10):809-818. DOI: 10.1111/j.1365-2591.2006.01155.x.

17. Albaladejo A, Osorio R, Papacchini F, et al. Post silanization improves bond strength of translucent posts to flowable composite resins. J Biomed Mater Res B Appl Biomater 2007;82(2):320-324. DOI: 10.1002/jbm.b.30736.

18. Goracci C, Sadek F, Ferrari M. Evaluation of the adhesion of fiber posts to intra-radicular dentin. Oper Dent 2005;30(5):627-635.

19. Cekic-Nagas I, Sukuroglu E, Canay S. Does the surface treatment affect the bond strength of various fibre-post systems to resin-core materials? J Dent 2011;39(2):171-179. DOI: 10.1016/j.jdent.2010. 11.008.

20. Cekic-Nagas I, Ergun G, Nagas E, et al. Comparison between regional micropush-out and microtensile bond strength of resin composite to dentin. Acta Odontol Scand 2008;66(2):73-81. DOI: 10.1080/00016350801958266.

21. Kienanen P, Alander P, Lassila LVJ, et al. Bonding of ceramic insert to a laboratory particle filler composite. Acta Odontol Scand 2005;63(5):272-277. DOI: 10.1080/00016350510020025.

22. Sadek F, Monticelli F, Goracci C, et al. Bond strength performance of different resin composites used as core materials around fiber posts. Dent Mater 2007;23(1):95-99. DOI: 10.1016/j.dental.2005 12.005.

23. Goracci C, Tavares A, Fabianelli A, et al. The adhesión between fiber posts and root canal walls: Comparison between microtensile and push-out bond strength measurements. Eur J Oral Sci 2004;112(4):353-361. DOI: 10.1111/j.1600-0722.2004.00146.x.

24. Vichi A, Grandini S, Davidson C, et al. An SEM evaluation of several adhesive systems used for bonding fiber posts under clinical conditions. Dent Mater 2012;18(7):495-502. DOI: 10.1016/S01095641(01)00065-3.

25. Chapell R, Cobb C, Spencer P, et al. Dentin tubule anastomosis: a potential factor in dentinal adhesive bonding? J Prosthet Dent 1994;72(2):183-188. DOI: 10.1016/0022-3913(94)90078-7.

26. Sahafi A, Peutzfeldt A, Asmusse E, et al. Bond strength of resin cement to dentin and to surface-treated posts of titanium alloy, glass fiber, and zirconia. J Adhes Dent 2003;5(2):153-162.

27. Cecchin D, Farina A, Vitti R, et al. Acid etching and surface coating of glass-fiber posts: bond strength and interface analysis. Braz Dent J 2016;27(2):228-233. DOI: 10.1590/0103-6440201600722.

28. Pyun J, Shin T, Lee J, et al. Effects of hydrogen peroxide pretreatment and heat activation of silane on the shear bond strength of fiberreinforced composite posts to resin cement. J Adv Prosthodont 2016;8(2):94-100. DOI: 10.4047/jap.2016.8.2.94.

29. Belwalkar V, Gade J, Mankar N. Comparison of the effect of shear bond strength with silane and other three chemical presurface treatments of a glass fiber-reinforced post on adhesión with a resin-based luting agent: an in vitro study. Contemp Clin Dent 2016;7(2):193-197. DOI: 10.4103/0976-237X.183056.

30. Archana $\mathrm{CH}$, Krishna Raju SM, Yadhav SS, et al. Effect of surface pretreatment and thermal Activation of silane coupling agent on bond strength of fiber posts to resin cement. J Contemp Dent Pract 2019;20(11):1293-1296. DOI: 10.5005/jp-journals-10024-2687. 